\title{
Simulating Thermal Noise for S/TEM Images from Atom Coordinate Data
}

\author{
Joseph Tessmer ${ }^{1}$, Marc De Graef ${ }^{1}$
}

${ }^{1}$ Dept. of Materials Science and Engineering, Carnegie Mellon Univ., Pittsburgh, PA, USA.

Scanning Transmission Electron Microscopy (STEM) is a powerful tool for characterizing materials. One method for characterization of materials by STEM is the comparison of simulated and experimental images, which can help identify the physical mechanism behind different contrast features. The presence of thermal noise represents a challenge for a direct comparison of simulated and experimentally obtained 4D-STEM images. Thermal noise arises because the captured image is the sum of contributions from many different electrons, each of which have passed through the sample. Each electron that interacts with the sample sees atoms approximately frozen in a random but correlated con-figuration; each electron samples a different atomic configuration and thus sees a modified electrostatic lattice potential [1]. In the past, the frozen phonon model has been used to simulate this thermal noise [2]. This model entails treating each atom as an independent Simple Harmonic Oscillator (SHO) with three orthogonal degrees of freedom, and generating multiple images for randomly sampled positions on the distribution for these oscillators, followed by averaging these images. This approach has the downside of requiring multiple simulation loops to produce a single image. Therefore, development of an algorithm that allows for inclusion of this thermal noise without requiring multiple simulation loops is desirable.

Previous work has demonstrated the ability to simulate STEM images from Molecular Dynamics (MD) atom coordinate data [3]. In this work, thermal noise was accounted for by using a DebyeWaller Fac-tor gathered from literature [4]. These Debye-Waller factors are constant for a given atomic number and sample temperature. Typically, the Debye-Waller factor is taken to be the same for every atom of a given type in the sample, regardless of whether this atom is located near a dislocation core or in a relatively unperturbed region of the sample. This leads to an incorrect accounting for thermal noise in the simulated image in regions near defects.

In this contribution, we introduce a new approach that makes use of both the Debye-Waller factor and the frozen phonon model, leading to increased computational efficiency while still reproducing atomic noise in a manner similar to the frozen phonon model. The Debye-Waller factor $B$ can be calculated by $B=8 \pi^{2}\left\langle u_{x}\right\rangle^{2}$, where $\left\langle u_{x}\right\rangle$ is the mean square displacement in the $x$ direction. Traditionally, this displacement value has been computed by using a harmonic approximation [4]. However, Molecular Dynamics simulations are sufficiently accurate to allow extraction of the mean displacement by averaging the displacement of each atom over multiple time steps. For validated interatomic potentials, this accurately reproduces the theoretically predicted Debye-Waller factor for sufficiently large sets of atoms (approximately 1, 000 atoms).

Rather than averaging the displacement over all atoms in the sample, we can instead save the mean square displacement for each atom individually and use this to compute a Single-Atom Debye-Waller Factor (SADWF). To ensure that the dataset for each atom is sufficiently large, we average the $\mathrm{x}, \mathrm{y}$, and $\mathrm{z}$ mean square displacements. When the image is simulated, rather than using a constant DWF for each atom type present in the sample, we use this SADWF. This leads to a similar distribution of pixel inten-sity, although the distribution for the single-atom DWF is skewed somewhat towards lower-intensity values. We have also found that when multiple MD snapshots are not available, a Monte Carlo sim- 
ulation can be used to obtain similar results by displacing each atom randomly in $\mathrm{x}, \mathrm{y}$, and $\mathrm{z}$ several times, provided that the mean of the normal distribution of displacements is the same as that predicted by the harmonic approximation. It is important to note that this approach to simulating thermal noise is not appropriate for atomic-scale imaging, where the phase shifts of the electron wavefunction due to the displacements of individual atoms are relevant.

Figure $1(\mathrm{a}-\mathrm{d})$ show the results for a $49 \times 49 \times 10$ unit cell molecular dynamics simulation of FCC Al single crystal at $300 \mathrm{~K}$, allowed to evolve over 25 time steps. 1(a) is the frozen phonon model image, the average of individual images computed at each time step. 1(b) is the SADWF image of the same dataset, where the mean displacements for each atom were calculated and a single-atom Debye Waller factor was used. 1(c) and 1(d) are the respective pixel intensity Kernel Density Estimation (KDE) functions. Figure 1(e-h) are similar, except a Monte Carlo simulation was used for the displacement calculations rather than a molecular dynamics simulation [5].

\section{References:}

[1] De Graef, Marc. Intro. to conventional transmission electron microscopy. Cambridge University Press, 2003.

[2] Loane, Russel F., PeiRong Xu, and John Silcox. Acta Crystallographica Section A: Foundations of Crystallography 47.3 (1991): 267-278.

[3] Tessmer, Joseph, Saransh Singh, and Marc De Graef. Microscopy and Microanalysis 24.S1 (2018), p. 208.

[4] Peng, L-M., et al. Acta Crys-tallographica Section A: Foundations of Crystallography 52.3 (1996), p. 456.

[5] The authors acknowledge an ONR Vannevar Bush Faculty Fellowship (N00014-16-1-2821), and the compu-tational resources of the Materials Characterization Facility at CMU, grant MCF-677785.

Molecular Dynamics

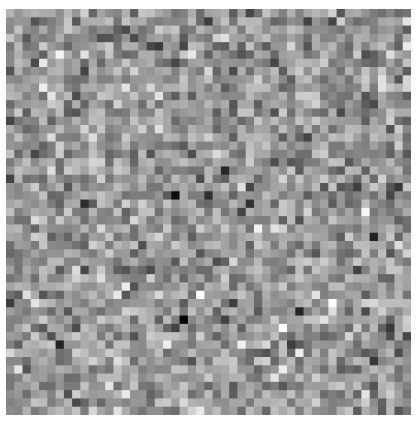

(a) Frozen Phonon

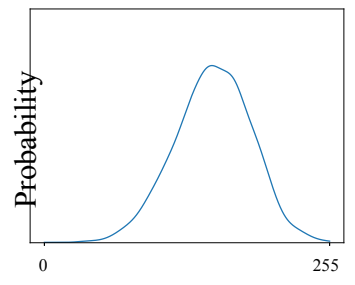

(c) $\mathrm{KDE}$

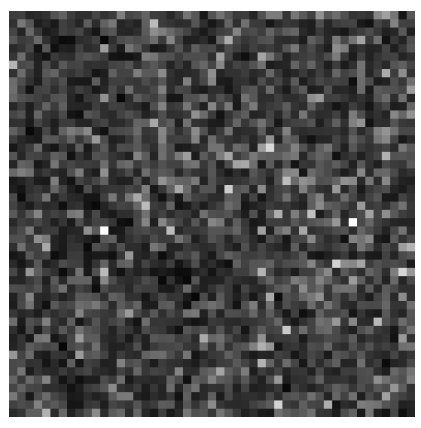

(b) SADWF

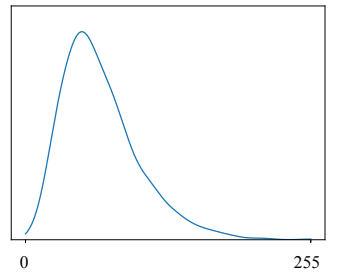

(d) $\mathrm{KDE}$

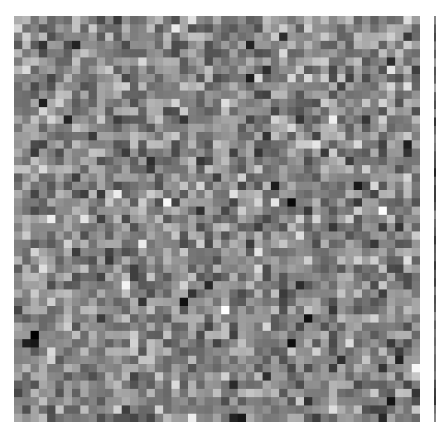

(e) Frozen Phonon

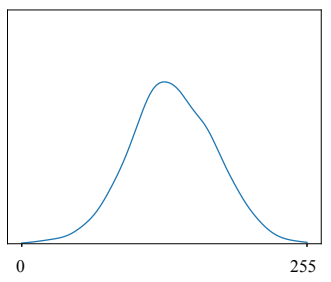

(g) KDE

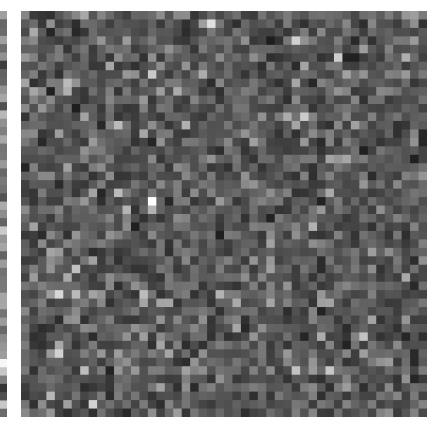

(f) SADWF

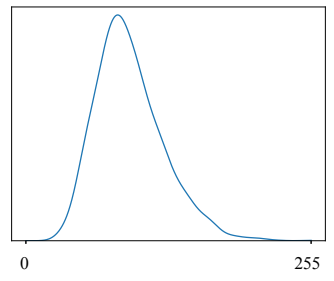

(h) KDE

Figure 1. Simulations of Thermal Noise in 4D-STEM Imaging. Top: Simulated ADF images for FCC Al. Bottom: Kernel density estimation for pixel intensity. 